\title{
Why plants need more than one type of auxin.
}

Sibu Simon and Jan Petrášek*

Institute of Experimental Botany, ASCR, Rozvojová 263, 16502 Praha 6, Czech Republic

\section{Corresponding author:}

Jan Petrášek

Institute of Experimental Botany ASCR, v. v. i.

Rozvojová 263

16502 Prague 6

Czech Republic

Phone: (+420) 225106429

Fax: (+420) 225106456

E-mail: petrasek@ueb.cas.cz 


\section{Abstract}

The versatile functionality and physiological importance of the phytohormone auxin is a major focus of attention in contemporary plant science. Recent studies have substantially contributed to our understanding of the molecular mechanisms underlying the physiological role of auxin in plant development. The mechanism of auxin action includes both fast responses not involving gene expression, possibly mediated by Auxin Binding Protein 1 (ABP1), and slower responses requiring auxin-regulated gene expression mediated by F-box proteins. These two mechanisms of action have been described to varying degrees for the major endogenous auxin indole-3-acetic acid (IAA) and for the synthetic auxins 2,4dichlorophenoxyacetic acid (2,4-D) and naphthalene-1-acetic acid (NAA). However, in addition to IAA, plants synthesize three other compounds that are commonly regarded as "endogenous auxins", namely, 4-chloroindole-3-acetic acid (4-Cl-IAA), indole-3-butyric acid (IBA) and phenylacetic acid (PAA). Although a spectrum of auxinic effects has been identified for all these as well as several other endogenous compounds, we remain largely ignorant of many aspects of their mechanisms of action and the extent to which they contribute to auxin-regulated plant development. Here, we briefly summarize the action of IBA, 4-Cl-IAA and PAA, and discuss the extent to which their action overlaps with that of IAA or results from their metabolic conversions to IAA. Other possible pathways for their action are considered. We present a scheme for homeostatic regulation of IAA levels that embraces other endogenous auxins in terms of the described mechanism of auxin action including its receptor and downstream signal transduction events.

Keywords : Auxin, IAA, 4-Cl-IAA, IBA, PAA 


\section{Contents}

1. Introduction: the elusive definitions of "auxin” and "auxin action”

2. IAA perception and signal transduction: transcriptional and non-transcriptional action

3. IAA in plant development: auxin gradients regulating morphogenesis

4. IBA: IAA precursor and a storage form

5. Other endogenous auxins: how many we might expect?

\subsection{4-Cl-IAA}

\subsection{PAA}

6. Auxin homeostasis: an interplay between IAA and other auxins?

7. Concluding remarks: closer, but still quite far from full understanding of auxin action Acknowledgements

References 


\section{Introduction: the elusive definitions of "auxin"and "auxin action"}

Studies of "auxins" originated in investigations of bending responses of coleoptiles towards a light source [1]. The signal perceived by the coleoptile tip was shown to be transported asymmetrically from the tip downward, where it induced growth response due to differential elongation of one side of the coleoptile [2]. This chemical signal was later identified as IAA $[3,4]$. The definition of “auxin activity” was thus initially based on the competence of substances to promote elongation growth in coleoptiles, but also to stimulate rooting [5].

Later research convincingly demonstrated that auxin is required together with other plant hormones for both cell division and oriented cell expansion [6, 7], influencing all aspects of plant development [8]. Consequently, it has become difficult to unambiguously define typical “auxin activity”. Auxin displays morphogenic properties [9] that are modulated by the environment and defined by dynamic changes in its perception and signal transduction. This machinery has been intensively studied during the past decade and includes effects that are either dependent or independent of gene expression. Thus “auxin action” may be understood as the sum of all these processes.

Many heterogeneous synthetic substances have auxin activity, complicating studies of structure-activity and the search for a common mode of action [10]. Even the most frequently used synthetic auxins, 2,4-dichlorophenoxy acetic acid (2,4-D) and naphthalene-1-acetic acid (NAA), do not completely share their mechanism of action with native IAA. Unlike IAA, 2,4D is not a good substrate for the auxin-binding protein ABP1 [11] and is poorly transported by auxin efflux carriers [12]. Only IBA [13], PAA [14] and 4-Cl-IAA [15] (Fig. 1) are synthesized by plants and therefore qualify as “endogenous auxins” [6], but their roles and mechanisms of action have not been satisfactorily described. The objective of this mini review is to summarize the present state of knowledge of the role of endogenous auxins and to highlight some of the uncertainties and unresolved questions related to their mechanism of action. 


\section{IAA perception and signal transduction: transcriptional and non-transcriptional action}

The identification of the nuclear auxin receptors of the TRANSPORT INHIBITOR RESPONSE 1/AUXIN SIGNALING F-BOX (TIR1/AFB) family of F-box proteins was a real breakthrough discovery in the field of auxin research $[16,17]$. These F-box proteins are subunits of SKP1 (S-phase kinase-associated protein 1)-Cullin-F-box protein (SCF) E3-ligase complex, which binds to auxin and is responsible for the degradation of Aux/IAA transcriptional repressors [18]. TIR1/AFB proteins are localized in the nucleus [19] and the mechanism of TIR1 binding to IAA, NAA and 2,4-D has been described in detail [20]. Auxin serves here as a kind of molecular glue that stabilizes the interaction between the receptor and Aux/IAA repressors of auxin response factors (ARFs), which are transcription factors that bind elements found in the promoters of many auxin-induced genes [21] (Fig. 2). Subsequent ubiquitination and proteasome-mediated degradation of these repressors facilitates the transcription of auxin-inducible genes [18]. The auxin response is complicated by the existence of five additional members of the AFB1-5 family [22, 23]. This mechanism, with various modifications, is common for the action of several other plant hormones as well as some environmental stimuli [24]. Interestingly, the lack of TIR1 or similar proteins responding to auxin in yeast and vertebrates allowed the generation of an auxin-based tool for efficient protein degradation in these organisms [25].

In light of recent discoveries of $\mathrm{SCF}^{\mathrm{TIR} 1 / \mathrm{AFBs}}$-mediated auxin-driven gene expression, all substances that bind to TIR1/AFBs or interfere with SCF ${ }^{\text {TIR1/AFBs }}$ pathway are sometimes being considered as auxins [26]. However, this interpretation of auxin action is undoubtedly too simplified because several very fast effects of auxin occur within seconds or minutes. Such responses are far too rapid to be explained by the effects of auxin on gene expression. One of the best described fast auxin effects is IAA-stimulated activity of plasma membrane $\mathrm{H}^{+}$-ATPases, followed by cell expansion due to the proton secretion and a decrease in apoplast $\mathrm{pH}$ (Fig. 2). The elevated $\mathrm{H}^{+}$release is compensated by an inward release of $\mathrm{K}^{+}$ions, which provides a positive turgor pressure for cell elongation and expansion [27, 28]. Auxin also increases cytosolic calcium concentration at non-genomic levels [29], activates reactive 
oxygen species [30] through the activation of phosphatidylinositol 3-kinase activity [31], and inhibits endocytosis [32].

Although still not unambiguously proved, a role of ABP1 has been suggested for some of the fast auxin responses connected with the activity of plasma membrane ion channels, cell expansion and endocytosis, as exhaustively reviewed recently [33]. There is still no convincing information about the downstream signaling pathway that is triggered following auxin binding to ABP1, but it appears likely that this protein is crucial for plant development since abp1 knockout mutants of Arabidopsis are embryo-lethal [34]. Interestingly, this mutation exhibits auxin transport-dependent phenotypes when maintained in heterozygous state and also shows changes in the expression of auxin-regulated genes [35], suggesting an indirect connection with an auxin signaling pathway. Another protein involved in the fast signaling pathway independent of SCF ${ }^{\text {TIR1/AFBs }}$ is the dual specifity phosphatase, INDOLE-3BUTYRIC ACID RESPONSE (IBR5) [36], which influences auxin-triggered fast increase of MAPK (mitogen-activating protein kinase) activity [37].

It seems that the overall action of IAA results from its fast non-transcriptional effects transduced probably by plasma membrane-located ABP1 or other intermediates $[33,38]$ and from its activation of gene expression by TIR1/AFBs. Exactly how these two mechanisms cooperate remains to be elucidated. The importance of ABP1 in the general auxin response is also strongly supported by the fact that the levels of AUX/IAAs dynamically reflect the down-regulation or over-expression of ABP1 [33], providing evidence for the functional coupling of ABP1 and SCF ${ }^{\mathrm{TIR} 1 / \mathrm{AFBs}}$ mechanisms. TIR1/AFBs-mediated expression may be also influenced by ABP1-triggered changes in the activity of plasma membrane phospholipases because IAA, NAA and 2,4-D elicit fast increase in phospholipase A2 activity [39] and inhibitors of this activity uncouple downstream SCF ${ }^{\text {TIR1/AFBs }}$ mediated gene expression [40]. Moreover, the auxin binding activity of TIR1/AFBs may be under the control of an important intermediate of plant phospholipid metabolism, inositol hexakisphosphate, which has its binding site located close to the pocket for IAA in TIR1 [20].

\section{IAA in plant development: auxin gradients regulating morphogenesis}


It is generally accepted that IAA regulates plant morphogenesis through tissue-specific concentration gradients that are formed by the processes of auxin biosynthesis, conjugation and degradation [41] as well as its intercellular [42] and intracellular distribution [43]. Here, IAA plays the role of a chemical transducer of a wide spectrum of external and internal signals. Undoubtedly, both transcriptional and non-transcriptional actions of IAA are involved. The concentration gradients of IAA in the tissues of the Arabidopsis thaliana model plant as well as other plants are important for embryogenesis [44], shoot and root organogenesis including apical dominance [45-47], vascular tissue development [48], and differential growth during tropisms and apical hook development [49]. IAA gradients may also be decisive in senescence [50], plant-pathogen interactions [51], abiotic stress responses [52] and other reactions of plants to the environment [53]. The morphogenic action of auxin is not always connected with its concentration maxima since auxin minimum has likewise been reported to regulate organogenesis during the opening of Arabidopsis thaliana fruits [54].

The establishment of IAA concentration gradients depends largely on IAA conjugation, de-conjugation, degradation and a regulated IAA transport across membranes. Although most of IAA is synthesized in young leaves, virtually all tissues of Arabidopsis thaliana seedlings synthesize IAA de novo [55]. IAA can be synthesized by both tryptophan-dependent and tryptophan-independent pathways, and resulting levels of free IAA are finely tuned by conjugation with amino acid, peptides or sugars $[41,56]$. The release of active IAA from these conjugates is under the control of hydrolytic enzymes [57].

Although biosynthesis and metabolism of IAA contribute significantly to the formation of auxin gradients, the transport of IAA represents the major determinant of differential distribution of auxin $[8,42]$. IAA is transported across the plasma membrane of individual cells either by passive diffusion of an undissociated (protonated) form (IAA) or by an active, carrier-mediated transport of a dissociated (acidic) form (IAA ${ }^{-}$). The more alkaline intracellular environment is characterized by a higher rate of IAA dissociation. Therefore, following a passive uptake of IAA into the cell, a significant amount of IAA ${ }^{-}$is trapped inside the cell and can only be transported out by an active carrier-mediated transport [58-60]. It is the subcellular localization of auxin influx and efflux transporters that determine the 
directionality of auxin transport [42]. Three types of active and mutually cooperating IAA transporting systems have been characterized in plants (Fig. 2). The PIN-FORMED (PIN) plasma membrane auxin efflux carriers $[61,62]$ are the primary determinants of the directionality of intercellular auxin flow. Based on indirect evidence, a subgroup of carriers containing PIN5, PIN6 and PIN8 was suggested to transport auxin between the endoplasmic reticulum, a compartment with IAA de-conjugation machinery [56], and the cytosol [43, 63]. The auxin influx carriers AUXIN RESISTANT 1/LIKE AUXIN RESISTANT (AUX1/LAX) $[64,65]$ and plant orthologs of the mammalian ATP-binding cassette subfamily B (ABCB)type transporters of the multidrug resistance/phosphoglycoprotein (ABCB/MDR/PGP) protein family $[66,67]$ are involved in generating local auxin sinks as well as containing auxin pools in order to sustain the main auxin streams.

\section{IBA: IAA precursor and a storage form}

Since its identification as an endogenous compound in several plant species [68, 69], IBA has been considered to be an endogenous auxin. IBA makes up as much as 25 to $30 \%$ of the total auxin present in Arabidopsis seedlings [70]. Although a possible IAA-independent pathway for IBA biosynthesis has been suggested [69], so far only one pathway has been demonstrated in which the side chain of pre-formed IAA is lengthened by the action of IBA synthase $[71,56]$. Therefore it is likely that the endogenous pool of IBA is entirely synthesized from IAA. IBA can be converted back to IAA by the action of peroxisomal âoxidation enzymes [72, 73] and may serve as a more stable [74] storage form of IAA [41, 56]. This compartmentation of IBA metabolism requires transporters that transport IBA into the peroxisomes. A good candidate for this role is the PEROXISOMAL ATP BINDING transporter (PXA1) of fatty acids, identified in a mutant screen for resistance to IBA [75]. The transport of IAA out of the peroxisomes may be an active process, but supporting data are lacking (Fig. 2).

IBA probably serves as an important source of IAA in tightly regulated developmental and environmental contexts. Formation of IAA from IBA in young Arabidopsis seedlings [76] plays a role in the elongation of root hairs and in the expansion of cotyledon cells, whereas 
hypocotyl elongation is maintained by the pool of IAA formed from IAA-amino acid conjugates [76]. The adaptation of IBA-IAA homeostasis is also influenced by hydrogen peroxide-stimulated production of IBA under drought and salinity stress, and these conditions are characterized by elevated levels of IBA and IAA and their conjugates [77]. Synthesis of IBA in maize roots is also induced by drought stress [71] and increased after infection with an arbuscular mycorrhizal fungus [78].

Importantly, IBA is actively transported in plants utilizing a transport machinery parallel to the well-characterized IAA carrier-mediated transport machinery [79]. Two candidate plasma membrane transporters from the ABCBG group of multidrug resistance proteins PLEIOTROPIC DRUG RESISTANCE (PDR) PDR8/ABCG36 [80] and PDR9/ABCBG37 [81, 82] mediate an active cellular efflux of IBA in Arabidopsis thaliana roots (Fig. 2). Both transporters have a broad specificity and transport a wide spectrum of auxinic compounds and auxin metabolites. Interestingly, however, they do not transport IAA itself. Therefore, in addition to being a storage form of IAA, IBA through its conversion to IAA may also play an important role in the generation of IAA concentration maxima during development or responses to stress.

An intriguing question still remains as to whether IBA can induce responses that are partially or completely independent of IAA. Currently available evidence argues against this possibility. First, because no IAA-independent biosynthetic pathway for IBA has yet been found, it seems that IBA levels depend on the levels of IAA. Second, there is no information to indicate that IBA is a substrate for either ABP1 or TIR1/AFBs. ABP1 levels increase after the colonization of maize roots with an arbuscular mycorrhizal fungus [78], suggesting that the ABP1 pathway is stimulated there. IBA also seems to weakly induce TIR1-mediated transcriptional activity of auxin-sensitive promoter elements in Arabidopsis seedlings [83], although this weak activity may easily be attributed to IAA.

Altogether, work over a number of years has shown that IBA serves as an important regulator of auxin activity, particularly during the formation of tissue specific IAA gradients. Its effects are tightly connected to IAA and IAA maxima, although it remains to be seen whether or not it has auxin activity in its own right. 


\section{Other endogenous auxins: how many we might expect?}

\subsection{4-Cl-IAA}

4-Cl-IAA is another endogenous auxin that is active in various auxin-related physiological assays and is often more active than IAA when exogenously applied [15, 84]. 4Cl-IAA has been identified as an endogenous auxin in several legumes and Pinus sylvestris [84], but to our knowledge not in Arabidopsis. It is usually found in developing seeds in those species, although there is also some evidence indicating it may occur in other tissues. Its biosynthesis is independent of IAA, instead originating from 4-Cl-Trp as a precursor. However, detailed information on the pathway or its alternatives is still missing [84]. The higher activity of 4-Cl-IAA could be due to its greater chemical stability than IAA [85] or to an unknown independent signaling pathway specific for this compound. Probably the most important physiological effect of 4-Cl-IAA is the stimulation of pericarp growth, which has been observed after exogenous application to de-seeded pea pericarp [86]. In contrast, application of IAA is either ineffective or inhibitory to this process [84]. Pea 4-Cl-IAA is known to be transported to the pericarp where it stimulates the production of bioactive gibberellins needed for fruit set and pericarp elongation [87, 88]. It remains to be determined what role IAA plays in this process and in particular whether there could be some analogy with the establishment of regulated IAA gradients that have been observed during later stages of Arabidopsis fruit development [54].

The mechanism of action of 4-Cl-IAA remains enigmatic, probably due to its absence in Arabidopsis. There is also complete lack of evidence whether 4-Cl-IAA has binding affinity for TIR1/AFBs, although the stimulation of auxin-responsive promoter element and 4-ClIAA-resistant growth in tir1 single mutant Arabidopsis seedlings has been observed [89]. Moreover, the binding affinity of 4-Cl-IAA to auxin binding sites correlates with its stimulatory effects on the elongation of maize coleoptiles [90]. Therefore, 4-Cl-IAA is probably involved in fast non-transcriptional responses in maize connected with proton secretion and the maintenance of membrane potential in coleoptiles [91] as well as in 4-ClIAA-induced protoplast swelling [92]. 


\subsection{PAA}

PAA is the only phenyl-derivative among the four endogenous auxins (Fig. 1). It usually exhibits weaker auxin effects in comparison with IAA, for example, in the case of elongating bean stems [93]. The presence of PAA was confirmed in various plant species and tissues in a wide concentration range [94]. The presence of nitrilase and nitrilase gene family in nasturtium suggested that biosynthesis of PAA uses the nitrilase pathway with a benzylglucosinolate precursor [95]. In addition to the auxin effects of PAA, root symbiotic bacteria produce PAA with anti-microbial activity [96, 97]. The biosynthesis of this bacterial PAA as well as that of IAA is under the feedback control of PAA itself and therefore it could be speculated that PAA preferentially plays a role in plant root interactions with soil microorganisms. Moreover, the fact that PAA inhibits carrier-mediated efflux of IAA in pea [98] suggests that it may be responsible for root-symbiont or root-pathogen interactions, integrating both auxin biosynthesis and transport. PAA binds strongly to ABP1 from corn [11] and also induces some conformational change in the structure of ABP1 [99], suggesting that the mechanism of action of PAA may include ABP1. However, data for the involvement of TIR1/AFBs in PAA signal transduction are completely lacking.

\section{Auxin homeostasis: an interplay between IAA and other auxins?}

As shown in this review, the so-called "endogenous auxins" form a group of compounds without a clear unifying mechanism of action. Because of the presence of IAA and IBA in the model plant Arabidopsis thaliana, we know much more about these two compounds than we do about the two other endogenous auxins, 4-Cl-IAA and PAA, which have not been detected in Arabidopsis. The mechanism of auxin action is complex and includes both fast effects occurring without the involvement of gene expression and rather slower effects requiring gene expression. The TIR1/AFBs-mediated pathway is active for IAA, but there are no data indicating that the action of the other three endogenous auxins operates through this pathway. The available evidence suggests that IBA is active only after conversion to IAA. So far suggestions for direct effects of 4-Cl-IAA and PAA remain hypothetical. The fast auxin 
effects that are transduced through the activation of ABP1 at the plasma membrane may be expected for IAA and 4-Cl-IAA, but are less likely in case of IBA and PAA. Homologs of ABP1 and IBR5, which are important for various fast auxin responses (see chapter 2), appeared early with the evolution of algae [100]. In comparison, the TIR1/AFBs-mediated pathway evolved later along with plant multicellularity and has become an important coordinating system in multicellular plants [100].

The complicated metabolism of IAA, which encompasses many enzyme systems involved in its activation and deactivation, has not been discussed in this review. Nevertheless, it is clear that homeostasis of free auxin pools in the plant plays a vital part in regulating auxin action in development and plant responses to environmental change. The endogenous auxin IBA contributes significantly to this free and hence active pool of IAA. It remains to be tested whether this applies also for the other two endogenous auxins.

Alternatively, 4-Cl-IAA seems to play a specialized role during the development of seeds and fruits in a limited range of species, whilst PAA possibly acts in co-ordination with IAA during plant interactions with soil microorganisms. It is likely that with more studies of IBA, PAA and 4-Cl-IAA, perhaps some other specific responses will be linked to each of these compounds and that their target receptor(s) will be identified.

\section{Concluding remarks}

It is clear from the observations reviewed here that defining "auxins" simply in terms of their ability to stimulate plant growth is no longer tenable. It is now apparent that IAA itself has wide-ranging and pleiotropic effects encompassing the regulation of almost every aspect of plant development, including cell growth, cell differentiation, histogenesis, organogenesis and responses to environmental changes. Abundant evidence indicates clear correlation between IAA concentration in a given tissue and the nature and magnitude of the response it invokes. This shifts the focus of investigations of auxin action toward mechanisms that regulate homeostasis of endogenous auxin pools in which processes such as synthesis, degradation, conjugation and specific transport play crucial roles, and toward mechanisms of action of IAA at the cellular and molecular/genomic level. 
From the point of view of terminology, it may be desirable to redefine the group of compounds termed “endogenous auxins” because their mechanisms of action may vary. However, several outstanding questions remain to be answered before we can fully understand the mechanisms that determine auxin homeostasis. First of all, the specificity of TIR1/AFBs receptors towards IBA, 4-Cl-IAA and PAA needs to be experimentally determined to exclude or confirm the binding of these auxins and their role, if any, in downstream proteasome-mediated gene expression. It is also necessary to determine how the fast non-transcriptional responses and those involving transcriptional regulation are coupled and how the signalling cascade triggered by ABP1 is constituted. The specificity of auxin transporters towards individual auxin-like compounds has been largely overlooked and requires further investigation, possibly by means of chemical or genomic approaches. An analysis of regulatory elements in the promoter regions of individual genes involved in auxin metabolism, signalling and transport may also yield valuable information.

\section{Acknowledgements}

This work was supported by the Ministry of Education, Youth and Sports of the Czech Republic, project LC06034. Authors would like to greatly acknowledge Dr. David Morris and Ass. Prof. Jan Marc for their critical reading. 


\section{References}

[1] C. Darwin, F. Darwin, The power of movement in Plants, D. Appleton and Co., New York, NY (1980).

[2] F. W. Went, Wuchsstoff und Wachstum, Rec. Trav. Bot. Neerland. 24 (1928) 1-116.

[3] F. Kögl, A. J Haagen-Smit, H. Erxleben, Über die neues auxin (heteroauxin) aus hain, XI Mitteilung. Zeitschr. Physiol. Chem. 228 (1934) 90-103.

[4] P. J. Davies, Plant hormones: biosynthesis, signal transduction, action! Springer (2004).

[5] F. W. Went, A test method for rhizocaline, the root forming substance, Proc. Kon. Akad. Wetenschap. Amsterdam. 37 (1934) 445-455.

[6] F. Skoog, C.O. Miller, Chemical regulation of growth and organ formation in plant tissue cultures in vitro, Symp. Soc. Exp. Biol. 11 (1957) 118-131.

[7] C. Perrot-Rechenmann, Cellular responses to auxin: division versus expansion, Cold Spring Harb. Perspect. Biol. 2 (2010) a001446.

[8] S. Vanneste, J. Friml, Auxin: a trigger for change in plant development, Cell 136 (2009) 1005-1016.

[9] R. P. Bhalerao, M. J. Bennett, The case for morphogens in plants, Nat. Cell. Biol. 5 (2003) 939-943.

[10] N. Ferro, T. Bredow, H. J. Jacobsen, T. Reinard, Route to Novel Auxin: Auxin chemical space toward biological correlation carriers, Chem. Rev. 110 (2010) 4690-4708.

[11] M. Löbler, D. Klämbt, Auxin-binding protein from coleoptile membranes of corn (Zea mays L.). I. Purification by immunological methods and characterization, J. Biol. Chem. 260 (1985) 9848-9853.

[12] A. Delbarre, P. Muller, V. Imhoff, J. Guern, Comparison of mechanisms controlling uptake and accumulation of 2,4-dichlorophenoxy acetic acid, naphthalene-1-acetic acid, and indole-3-acetic acid in suspension-cultured tobacco cells, Planta 198 (1996) 532-541.

[13] P. W. Zimmerman, F. Wilcoxon, Several chemical growth substances which cause initiation of root and other responses in plants, Contr. Boyce Thompson Inst. 7 (1935) 209. 
[14] J. B. Koepfli, K.V. Thimann, F. W. Went, Phytohormones: structure and physiological activity. É, J. Biol. Chem. 122 (1938) 763-780.

[15] W. L. Porter, K. V. Thimann, Molecular requirements for auxin action-I. Halogenated indoles and indoleacetic acid, Phytochemistry 4 (1965) 229-243.

[16] N. Dharmasiri, S. Dharmasiri, M. Estelle, The F-box protein TIR1 is an auxin receptor, Nature 435 (2005) 441-445.

[17] S. Kepinski, O. Leyser, The Arabidopsis F-box protein TIR1 is an auxin receptor, Nature 435 (2005) 446-451.

[18] W. M. Gray, S. Kepinski, D. Rouse, O. Leyser, M. Estelle, Auxin regulates SCF ${ }^{\mathrm{TIR}}$ dependent degradation of AUX/IAA proteins, Nature 414 (2001) 271-276.

[19] N. Dharmasiri, S. Dharmasiri, D. Weijers, E. Lechner, M. Yamada, L. Hobbie, J. S. Ehrismann, G. Jürgens, M. Estelle, Plant development is regulated by a family of auxin receptor F box proteins, Dev. Cell 9 (2005) 109-119.

[20] X. Tan, L. I. Calderon-Villalobos, M. Sharon, C. Zheng, C. V. Robinson, M. Estelle, N. Zheng, Mechanism of auxin perception by the TIR1 ubiquitin ligase, Nature 446 (2007) 640-645.

[21] T. Ulmasov, G. Hagen, T. J. Guilfoyle, Activation and repression of transcription by auxin-response factors. Proc. Natl. Acad. Sci. U.S.A. 96 (1999) 5844-5849.

[22] G. Parry, L. I. Calderon-Villalobos, M. Prigge, B. Peret, S. Dharmasiri, H. Itoh, E. Lechner, W. M. Gray, M. Bennett, M. Estelle, Complex regulation of the TIR1/AFB family of auxin receptors, Proc. Natl. Acad. Sci. U. S. A. 106 (2009) 22540-22545.

[23] T. A. Walsh, R. Neal, A.O. Merlo, M. Honma, G. R. Hicks, K. Wolff, W. Matsumura, J. P. Davies, Mutations in an auxin receptor homolog AFB5 and in SGT1b confer resistance to synthetic picolinate auxins and not to 2,4-dichlorophenoxyacetic acid or indole-3-acetic acid in Arabidopsis, Plant. Physiol. 142 (1996) 542-552.

[24] R. D. Vierstra, The ubiquitin-26s proteasome system at the nexus of plant biology, Nat. Rev. Mol. Cell. Biol. 10 (2009) 385-397. 
[25] K. Nishimura, T. Fukagawa, H. Takisawa, T. Kakimogto, M. Kanemaki, An auxin-based degron system for the rapid depletion of proteins in non plant cells, Nat. Methods 6 (2009) 917-922.

[26] B. De Rybel, D. Audenaert, T. Beeckman, T. Kepinski, The past, present and future of chemical biology in auxin research, ACS Chem. Biol. 4 (2009) 987-998.

[27] A. Hager, G. Debus, H. G. Edel, H. Stransky, R. Serrano, Auxin induces exocytosis and the rapid synthesis of a high-turnover pool of plasma membrane $\mathrm{H}^{+}$-ATPase, Planta 185 (1991) 527-537.

[28] A. Hager, Role of the plasma membrane $\mathrm{H}^{+}$-ATPase in auxin-induced elongation growth: historical and new aspects, J. Plant Res. 116 (2003) 483-505.

[29] M. Shishova, S. Lindberg, Auxin induces an increase of $\mathrm{Ca}^{2+}$ concentration in the cytosol of wheat leaf protoplasts, J. Plant. Physiol. 161 (2004) 937-945.

[30] P. Schopfer, A. Liszkay, Plasma membrane-generated reactive oxygen intermediates and their role in cell growth of plants, Biofactors 28 (2006) 73-81.

[31] J. H. Joo, H. J. Yoo, I. Hwang, J. S. Lee, K. H. Nam, Y. S. Bae, Auxin-induced reactive oxygen species production requires the activation of phosphatidylinositol 3-kinase, FEBS Lett. 579 (2005) 1243-1248.

[32] T. Paciorek, E. Zažímalová, N. Ruthardt, J. Petrášek, Y. D. Stierhof, J. Kleine-Vehn, D. A. Morris, N. Emans, G. Jürgens, N. Geldner, J. Friml, Auxin inhibits endocytosis and promotes its own efflux from cells, Nature 435(2005) 1251-1256.

[33] A. Tromas, I. Paponov, C. Perrot-Rechenmann, AUXIN BINDING PROTEIN 1: functional and evolutionary aspects, Trends Plant Sci. 15 (2010) 436-446.

[34] J. Chen, H. Ullah, J. Young, M. Sussman, A. Jones, ABP1 is required for organized cell elongation and division in Arabidopsis embryogenesis. Genes Dev. 15 (2001) 902-911.

[35] Y. Effendi, S. Rietz, U. Fischer, G. F. E. Scherer, The heterozygous abp1/ABP1 insertional mutant has defects in functions requiring polar auxin transport and in regulation of early auxin-regulated genes. Plant J. (2010) DOI: 10.1111/j.1365313X.2010.04420.x. 
[36] L. C. Strader, M. Monroe-Augustus, B. Bartel, The IBR5 phosphatase promotes Arabidopsis auxin responses through a novel mechanism distinct from TIR1-mediated repressor degradation, BMC Plant Biol. 8 (2008) 41.

[37] J. S. Lee, S. Wang, S. Sritubtim, J. G. Chen, B. E. Ellis, Arabidopsis mitogen-activated protein kinase MPK12 interacts with the MAPK phosphatase IBR5 and regulates auxin signaling, Plant J. 57 (2009) 975-985.

[38] M. Shishova, S. Lindberg, A new perspective on auxin perception, J. Plant Physiol. 167 (2010) 417-422.

[39] R. Paul, A. Holk, G. F. E. Scherer, Fatty acids and lysophospholipids as potential second messengers in auxin action. Rapid activation of phospholipase A2 activity by auxin in suspension-cultured parsley and soybean cells, Plant J. 16 (1998) 601-611.

[40] G. F. E. Scherer, M. Zahn, J. Callis, A. M. Jones, A role for phospholipase A in auxinregulated gene expression, FEBS Lett. 581 (2007) 4205-4211.

[41] J. Normanly, Approaching cellular and molecular resolution of auxin biosynthesis and metabolism, Cold Spring Harb. Perspect. Biol. 2 (2010) a001594.

[42] J. Petrášek, J. Friml, Auxin transport routes in plant development, Development 136 (2009) 2675-2688.

[43] J. Mravec, P. Skùpa, A. Bailly, K. Hoyerová, P. Køeèek, A. Bielach, J. Petrášek, J. Zhang, V. Gaykova, Y. D. Stierhof, P. I. Dobrev, K. Schwarzerová, J. Rolèík, D. Seifertová, C. Luschnig, E. Benková, E. Zažímalová, M. Geisler, J. Friml, ER-localized PIN5 auxin transporter mediates subcellular homeostasis of phytohormone auxin, Nature 439 (2009) 1136-1140.

[44] J. Friml, A. Vieten, M. Sauer, D. Weijers, H. Schwarz, T. Hamann, R. Offringa, G. Jürgens, Efflux-dependent auxin gradients establish the apical-basal axis of Arabidopsis, Nature 426 (2003) 147-153.

[45] E. Benková, M. Michniewicz, M. Sauer, T. Teichmann, D. Seifertová, G. Jürgens, J. Friml, Local, efflux-dependent auxin gradients as a common module for plant organ formation, Cell 15 (2003) 591-602. 
[46] D. Reinhardt, T. Mandel, C. Kuhlemeier, Auxin regulates the initiation and radial position of plant lateral organs, Plant Cell 12 (2000) 507-518.

[47] J. G. Dubrovsky, M. Sauer, S. Napsucialy-Mendivil, M. G. Ivanchenko, J. Friml, S. Shishkova, J. Celenza, E. Benková, Auxin acts as a local morphogenetic trigger to specify lateral root founder cells, Proc. Natl. Acad. Sci. USA 105 (2008) 8790-8794.

[48] J. Mattsson, W. Ckurshumova, T. Berleth, Auxin signaling in Arabidopsis leaf vascular development, Plant Physiol. 131 (2003) 1327-1339.

[49] J. Friml, J. Wiœniewska, E. Benková, K. Mendgen, K. Palme, Lateral relocation of auxin efflux regulator PIN3 mediates tropism in Arabidopsis, Nature 415 (2002) 806-809.

[50] C. M. Ellis, P. Nagpal, J. C. Young, G. Hagen, T. J. Guilfoyle, J. W. Reed, AUXIN RESPONSE FACTOR1 and AUXIN RESPONSE FACTOR2 regulate senescence and floral organ abscission in Arabidopsis thaliana, Development 132 (2005) 4563-4574.

[51] Kazan K, Manners JM, Linking development to defense: auxin in plant-pathogen interactions, Trends Plant. Sci. 14 (2009) 373-382.

[52] S. Wang, Y. Bai, C. Shen, Y. Wu, S. Zhang, D. Jiang, T. J. Guilfoyle, M. Chen, Y. Qi, Auxin-related gene families in abiotic stress response in Sorghum bicolor, Funct. Integr. Genomics 10 (2010) 533-546.

[53] J. W. Chandler, Local auxin production: a small contribution to a big field, Bioessays 31 (2009) 60-70.

[54] K. Sorefan, T. Girin, S. J. Liljegren, K. Ljung, P. Robles, C. S. Galván-Ampudia, R. Offringa, J. Friml, M.F.Yanofsky, L. Østergaard, A regulated auxin minimum is required for seed dispersal in Arabidopsis, Nature 459 (2009) 583-586.

[55] K. Ljung, R. P. Bhalerao, G. Sandberg, Sites and homeostatic control of auxin biosynthesis in Arabidopsis during vegetative growth, Plant J. 28 (2001) 465-474.

[56] A. W. Woodward, B. Bartel, Auxin: regulation, action, and interaction, Ann Bot. 95 (2005) 707-735.

[57] R. A. Rampey, S. LeClere, M. Kowalczyk, K. Ljung, G. Sandberg, B. Bartel, A family of auxin-conjugate hydrolases that contributes to free indole-3-acetic acid levels during Arabidopsis germination, Plant Physiol. 135 (2004) 978-988. 
[58] P. H Rubery, A. R. Sheldrake, Carrier-mediated auxin transport, Planta 118 (1974) 101121.

[59] J. A. Raven, Transport of indoleacetic acid in plant-cells in relation to $\mathrm{pH}$ and electrical potential gradients, and its significance for polar IAA transport, New Phytol. 74 (1975) 163-172.

[60] M. H. M. Goldsmith, Polar transport of auxin, Annu. Rev. Plant Physiol. Plant Mol. Biol. 28 (1977) 439-478.

[61] L. Gälweiler, C. Guan, A. Müller, E. Wisman, K. Mendgen, A. Yephremov, K. Palme, Regulation of polar auxin transport by AtPIN1 in Arabidopsis vascular tissue, Science 282 (1998) 2226-2230.

[62] P. Køeèek, P. Skùpa, J. Libus, S. Naramoto, R. Tejos, J. Friml, E. Zažímalová, The PINFORMED (PIN) protein family of auxin transporters, Genome Biol. 10 (2009) 249.

[63] A. Ganguly, S. H. Lee, M. Cho, O. R. Lee, H. Yoo, H. T. Cho, Differential auxintransporting activities of PIN-FORMED proteins in Arabidopsis root hair cells, Plant Physiol. 153 (2010) 1046-1061.

[64] M. J. Bennett, A. Marchant, H. G. Green, S. T. May, S. P. Ward, P. A. Millner, A. R. Walker, B. Schulz, K. A. Feldmann, Arabidopsis AUX1 gene: a permease-like regulator of root gravitropism, Science 273 (1996) 948-950.

[65] K. Swarup, E. Benková, R. Swarup, I. Casimiro, B. Peret, Y. Yang, G. Parry, E. Nielsen, I. De Smet, S. Vanneste, M. P. Levesque, D. Carrier, N. James, V. Calvo, K. Ljung, E. Kramer, R. Roberts, N. Graham, S. Marillonnet, K. Patel, J. D. Jones, C. G. Taylor, D. P. Schachtman, S. May, G. Sandberg, P. Benfey, J. Friml, I. Kerr, T. Beeckman, L. Laplaze, M. J. Bennett, The auxin influx carrier LAX3 promotes lateral root emergence, Nat. Cell Biol. 10 (2008) 946-954.

[66] B. Noh, A. S. Murphy, E. P. Spalding, Multidrug resistance-like genes of Arabidopsis required for auxin transport and auxin-mediated development, Plant Cell 13 (2001) 24412454.

[67] M. Geisler, A. S. Murphy, The ABC of auxin transport: the role of p-glycoproteins in plant development, FEBS Lett. 580 (2006) 1094-1102. 
[68] J. Ludwig-Müller, E. Epstein, Occurrence and in vivo biosynthesis of indole-3-butyric acid in corn, Plant Physiol. 97 (1991) 765-770.

[69] J. Ludwig-Müller, Indole-3-butyric acid in plant growth and development, Plant Growth Regul. 32 (2000) 219-230.

[70] J. Ludwig Müller, S. Sass, E. G. Sutter, M. Wodner, E. Epstein, Indole-3-butyric acid in Arabidopsis thaliana I. Identification and quantification, Plant Growth Regul. 13 (1993) 179-187.

[71] J. Ludwig-Müller, W. Hilgenberg, Characterization and partial purification of indole-3butyric acid synthetase from maize (Zea mays), Physiol. Plant. 94 (1995) 651-660.

[72] E. Epstein, J. Ludwig Müller, Indole-3-butyric acid in plants: occurrence, synthesis, metabolism and transport, Physiol. Plant. 88 (1993) 382-389.

[73] B. K. Zolman, N. Martinez, A. Millius, A. R. Adham, B. Bartel, Identification and characterization of Arabidopsis indole-3-butyric acid response mutants defective in novel peroxisomal enzymes, Genetics 180 (2008) 237-251.

[74] A. C. Nordström, F. A. Jacobs, L. Eliasson, Effect of exogenous indole-3-acetic acid and indole-3-butyric acid on internal levels of the respective auxins and their conjugation with aspartic acid during adventitious root formation in pea cuttings, Plant Physiol. 96 (1991) 856-861.

[75] B. K. Zolman, I. D. Silva, B. Bartel, The Arabidopsis pxa1 mutant is defective in an ATP-binding cassette transporter-like protein required for peroxisomal fatty acid âoxidation, Plant Physiol. 127 (2001) 1266-1278.

[76] L. C. Strader, A. H. Culler, J. D. Cohen, B. Bartel, Conversion of endogenous indole-3butyric acid to indole-3-acetic acid drives cell expansion in Arabidopsis seedlings, Plant Physiol. 153 (2010) 1577-1586.

[77] V. B. Tognetti, O. Van Aken, K. Morreel, K. Vandenbroucke, B. van de Cotte, I. De Clercq, S. Chiwocha, R. Fenske, E. Prinsen, W. Boerjan, B. Genty, K. A. Stubbs, D. Inzé, F. Van Breusegem, Perturbation of indole-3-butyric acid homeostasis by the UDPglucosyltransferase UGT74E2 modulates Arabidopsis architecture and water stress tolerance, Plant Cell 22 (2010) 2660-79. 
[78] M. Kaldorf, J. Ludwig-Muller, AM fungi might affect the root morphology of maize by increasing indole-3-butyric acid biosynthesis, Physiol. Plant. 109 (2000) 58-67.

[79] A. M. Rashotte, J. Poupart, C. S. Waddell, G. K. Muday, Transport of the two natural auxins, indole-3-butyric acid and indole-3-acetic acid, in Arabidopsis, Plant Physiol. 133 (2003) 761-772.

[80] L. C. Strader, B. Bartel, The Arabidopsis PLEIOTROPIC DRUG RESISTANCE8/ABCG36 ATP binding cassette transporter modulates sensitivity to the auxin precursor indole-3-butyric acid, Plant Cell 21 (2009) 1992-2007.

[81] L. C. Strader, M. Monroe-Augustus, K. C. Rogers, G. L. Lin, B. Bartel, Arabidopsis iba response 5 suppressors separate responses to various hormones, Genetics 180 (2008) 2019-2031.

[82] K. Rùžièka, L. C. Strader, A. Bailly, H. Yang, J. Blakeslee, L. Langowski, E. Nejedlá, H. Fujita, H. Itoh, K. Syono, J. Hejátko, W. M. Gray, E. Martinoia, M. Geisler, B. Bartel, A. S. Murphy, J. Friml, Arabidopsis PIS1 encodes the ABCG37 transporter of auxinic compounds including the auxin precursor indole-3-butyric acid, Proc. Natl. Acad. Sci. U S A 107 (2010) 10749-10753.

[83] Y. Oono, Q. G. Chen, P. J. Overvoorde, C. Köhler, A. Theologis, Age mutants of Arabidopsis exhibit altered auxin-regulated gene expression, Plant Cell 10 (1998) 16491662.

[84] D. M. Reinecke, 4-Chloroindole-3-acetic acid and plant growth, Plant Growth Regul. 27 (1999) 3-13.

[85] S. Marumo, H. Hattori, A. Yamamoto, Biological activity of 4-chloroindolyl-3-acetic acid, Plant Growth Substances. Hirokawa Publishing Co. (1973) 419-428.

[86] D. M. Reinecke, J. A. Ozga, V. Magnus, Effect of halogenated substitution of indole-3acetic acid on biological activity of pea fruit, Phytochemistry 40 (1995) 1361-1366.

[87] J. A. Ozga, J. Yu, D. M. Reinecke, Pollination, development, and auxin-specific regulation of gibberellin 3 beta-hydroxylase gene expression in pea fruit and seeds, Plant Physiol. 131 (2003) 1137-1146. 
[88] J. A. Ozga, D. M. Reinecke, B. T. Ayele, P. Ngo, C. Nadeau, A. D. Wickramarathna, Developmental and hormonal regulation of gibberellin biosynthesis and catabolism in pea fruit, Plant Physiol. 150 (2009) 448-462.

[89] S. Simon, M. Kubeš, J. Petrášek, E. Zažímalová, Correlation between polar auxin transport specificity and auxin signalling, FEBS J. 276 (2009) (Suppl. 1) p. 252.

[90] U. Rescher, A. Walther, C. Schiebl and D. Klämbt, In vitro binding affinities of 4-chloro, 2-methyl-, 4-methyl-, and 4-ethylindoleacetic acid to auxin-binding protein 1 (ABP1) correlate with their growth-stimulating activities, J. Plant Growth Regul. 15 (1996) 1-3.

[91] W. Karcz, Z. Burdach, A comparison of the effects of IAA and 4-Cl-IAA on growth, proton secretion and membrane potential in maize coleoptile segments, J. Exp. Bot. 53 (2002) 1089-1098.

[92] B. Steffens, H. Luthen, New methods to analyse auxin induced growth II: the swelling reaction of protoplasts - a model system for the analysis of auxin signal transduction?, J. Plant Growth Regul. 32 (2000) 115-122.

[93] D. K. Small, D. A. Morris, Promotion of elongation and acid invertase activity in Phaseolus vulgaris L. internode segments by phenylacetic acid, J. Plant Growth Regul. 9 (1990) 329-340.

[94] F. Wightman, D. L. Lightly, Identification of phenylacetic acid as a natural auxin in the shoots of higher plants, Physiol. Plant. 55 (1982) 17-24.

[95] J. Ludwig-Müller, J. D. Cohen, Identification and quantification of three active auxins in different tissues of Tropaeolum majus, Physiol. Plant. 115 (2002) 320-329.

[96] P. J. Slininger, K. D. Burkhead, D. A. Schisler, Antifungal and sprout regulatory bioactivities of phenylacetic acid, indole-3-acetic acid, and tyrosol isolated from the potato dry rot suppressive bacterium Enterobacter cloacae, J. Ind. Microbiol. Biotechnol. 31 (2004) 517-524.

[97] E. Somers, D. Ptacek, P. Gysegom, M. Srinivasan, J. Vanderleyden, Azospirillum brasilense produces the auxin-like phenylacetic acid by using the key enzyme for indole3-acetic acid biosynthesis, Appl. Environ. Microbiol. 71 (2005) 1803-1810. 
[98] D. A. Morris, C. F. Johnson, Regulation of auxin transport in pea (Pisum sativum L.) by phenylacetic acid: inhibition of polar auxin transport in intact plants and stem segments, Planta 172 (1987) 408-416.

[99] R. M. Napier, M. A. Venis, Monoclonal antibodies detect an auxin-induced conformational change in the maize auxin-binding protein, Planta 182 (1990) 313-318.

[100] S. Lau, N. Shao, R. Bock, G. Jürgens, I. De Smet, Auxin signaling in algal lineages: fact or myth? Trends Plant Sci. 14 (2009) 182-188. 


\section{Figure legends}

Fig. 1. Chemical structure of four endogenous auxins. Indole-3-acetic acid (IAA), indole-3butyric acid (IBA), 4-chloroindole-3-acetic acid (4-Cl-IAA) and phenylacetic acid (PAA).

Fig. 2. Schematic depiction of the intracellular distribution and mechanisms of action for endogenous auxins. Besides passive diffusion of IAA, this major endogenous auxin utilizes AUX1/LAX influx carriers for the active uptake into the cell and PIN/ABCB transporters for the active efflux (carrier-mediated transport applies to IAA ${ }^{-}$). Nucleus-located auxin receptors from TIR1/AFB family of F-box proteins interact with auxin to initiate ubiquitin-mediated degradation of Aux/IAA auxin responsive gene repressors in order to activate auxin responsive genes [42]. The specifity of TIR1 for binding of 4-Cl-IAA, PAA and IBA has not been unfortunately tested yet. Plasma membrane located ABP1 is associated with an unknown docking protein and upon binding to auxin, ABP1 activates $\mathrm{H}^{+}$ATPase and potassium channel. The increase of $\mathrm{K}^{+}$ion causes the inflow of water into the cell and results in the cell expansion [33]. Major portion of ABP1 is localized in the lumen of endoplasmic reticulum, although the function of ABP1 is questionable there. It could perhaps cooperate with auxin transporters PIN5, PIN6 and PIN8 $[43,63]$ to transport IAA into and out of the ER, the compartment with conjugation and deconjugation machinery [56]. 4-Cl-IAA also seems to partially use the same transporters for active influx as IAA [89]. IBA is transported to peroxisomes using PXA1 ABC transporter and converted to IAA through peroxisomal âoxidation [72, 74]. It is also transported out of the cell through laterally-localized PDR transporters [80-82]. PAA transport across membrane seems not to be mediated by any carrier. It is rather reported to function as a regulator of IAA transport [98]. PAA's independent role as an auxin yet to be determined. See the main text for other details. 
<smiles>O=C(O)Cc1c[nH]c2ccccc12</smiles>

Indole-3-acetic acid

(IAA)<smiles>O=C(O)CCCc1c[nH]c2ccccc12</smiles>

Indole-3-butyric acid

(IBA)<smiles>Cc1c[nH]c2cccc(Cl)c12</smiles>

4-chloroindole-3-acetic acid

(4-Cl-IAA)<smiles>O=C(O)Cc1ccccc1</smiles>

Phenylacetic acid

(PAA) 


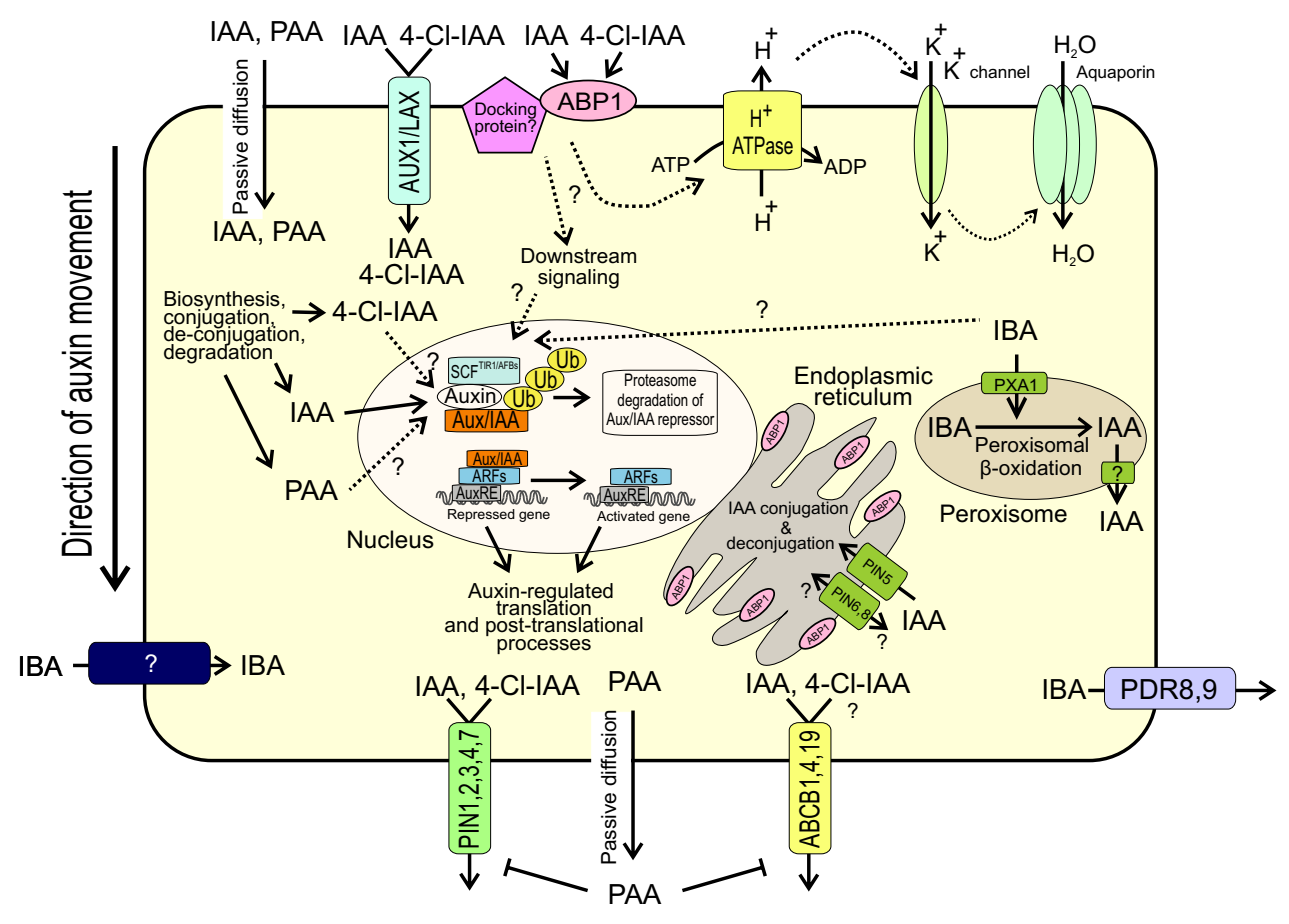

